\title{
Adipose-Derived Mesenchymal Stem Cell-Derived Exosomal miR-30la-3p Regulates Airway Smooth Muscle Cells During Asthma by Targeting STAT3
}

\author{
Chen-Ye Feng* \\ Shi-Yao Bai* \\ Meng-Lu Li \\ Jie-Yu Zhao \\ Jia-Min Sun \\ Hui-Jing Bao \\ Yuan Ren \\ Xin-Ming Su
}

Department of Pulmonary and Critical Care Medicine, Institute of Respiratory Diseases, The First Affiliated Hospital of China Medical University; Respiratory Disease Institution of China Medical University, Shenyang, Liaoning, People's Republic of China

*These authors contributed equally to this work
Correspondence: Xin-Ming Su Department of Pulmonary and Critical Care Medicine, Institute of Respiratory Diseases, The First Affiliated Hospital of China Medical University; Respiratory Disease Institution of China Medical University, 155 North Nanjing Street, Shenyang, I I000I, Liaoning, People's Republic of China

Email xinming_med@I26.com
Background: Asthma is a chronic inflammatory disease featured by inflammation and remodeling of airway. Adipose-derived mesenchymal stem cell (ADSCs)-derived exosomal miRNAs have been suggested as promising therapeutic manners for diseases.

Methods: ADSCs and airway smooth muscle cells (ASMCs) were isolated from SD rats. Flow cytometry was conducted to detect the surface biomarkers of isolated cells. Exosomes were extracted by sequentially centrifuge method and identified by Western blotting and nanoparticle tracking analysis (NTA). Uptake of exosomes by ASMCs was detected by confocal assay. ASMCs were treated with platelet-derived growth factor-BB (PDGF-BB) to mimic cell remodeling and inflammation. Cell counting 8 (CCK-8), Transwell, and flow cytometry were performed to determine the viability, migration, and apoptosis of ASMCs. Release of inflammatory factors was detected by enzyme-linked immunosorbent assay (ELISA). Levels of RNAs and proteins were measured by quantitative reverse transcription polymerase chain reaction (qRT-PCR) assay. Interaction between miR-301a-3p and signal transducer and activator of transcription 3 (STAT3) was determined by luciferase reporter gene assay. The effect of Exosomal miR-301a-3p was analyzed in ovalbumin (OVA)-induced asthma mouse model.

Results: ADSCs-derived exosomes could be effectively internalized by ASMCs. Exosomal miR-301a-3p notably suppressed the PDGF-BB-stimulated proliferation and migration of ASMCs, and enhanced apoptosis, as well as decreased the secretion of inflammatory factors. MiR-301a-3p directly targeted the 3'UTR region of STAT3. STAT3 overexpression reversed the suppressive effects of exosomal miR-301a-3p on ASMCs under PDGF-BB stimulation. The expression of miR-301a-3p and STAT3 was negative correlation in specimen from patients with asthma. Exosomal miR-301a-3p inhibited OVA-induced lung injury by targeting STAT3 in mice.

Conclusion: This study exposed that exosomal miR-301a-3p from ADSCs could effectively alleviate PDGF-BB-stimulated remodeling and inflammation of ASMCs via targeting STAT3, presented ADSCs-derived exosomal miR-301a-3p as a promising therapeutic approach for asthma.

Keywords: asthma, mesenchymal stem cell, exosome, miR-301a-3p, STAT3

\section{Introduction}

Asthma is a chronic inflammatory disease of respiratory system, which bothers the normal life of around 5\% adults and 10\% children, and presents an increasing incidence worldwide. ${ }^{1-3}$ Asthma is featured by allergic remodeling, inflammation, and hyperresponsiveness in airway. ${ }^{4,5}$ A thorough understanding of the mechanisms 
involved in these processes is hence of great importance for therapy of asthma. Recent studies have suggested the critical role of airway smooth muscle cells (ASMCs) during progression of asthma. ${ }^{6-8}$ ASMCs are the major component of airway, and are closely correlated with airway remodeling and inflammatory responses during asthma, manifested by abnormal levels of cytokines such as platelet-derived growth factor (PDGF). ${ }^{9,10}$ As an important member of PDGF, PDGF-BB has been widely suggested to stimulate the remodeling of airway via promoting proliferation and migration of ASMCs. ${ }^{11}$ Therefore, ameliorating PDGF-BB-induced airway inflammation and remodeling may represent a potential therapeutic approach for asthma.

Mesenchymal stem cells (MSCs) are a group of cells commonly derived from bone marrow and some other tissues such as adipose. ${ }^{12}$ Increasing number of evidences have proved the great potential of MSCs for disease therapy, owing to their low immunogenicity, ability of selfrenewal and differentiation. ${ }^{13}$ Recent studies even proposed that MSCs are involved in immune regulation. ${ }^{13}$ The MSCs exhibit their functions through paracrine secretion of various cytokines and chemokines or release of extracellular vesicles. ${ }^{12}$ Exosomes belong to extracellular vesicles and are capable of delivering mRNA, proteins, lipid, as well as microRNAs (miRNAs). ${ }^{14}$ Adiposederived mesenchymal stem cells (ADSCs) are also capable of secreting exosomes to facilitates cell communication and various cell functions. ${ }^{15-17}$ For example, ADSCsderived exosomal miR-181-5p suppresses autophagy of hepatic stellate cells and attenuated cell injury during liver fibrosis. ${ }^{18}$ MiR-301a-3p is a recently reported miRNA that is highly expressed in exosomes-derived from hypoxic pancreatic cells, and causes the M2 polarization of macrophages through regulating the PI3K signaling. ${ }^{19}$ Exosomal miR-301a-3p is also involved in the progression and metastasis of gastric cancer. ${ }^{20}$ Yet, the role of exosomal miR-301a-3p in asthma is still unclear.

Signal transducer and activator of transcription 3 (STAT3) is an important transcriptional factor involved in the development and mature of vertebrate tissues, as well as controlling inflammatory response and immunity. ${ }^{21}$ Study on asthmatic mice model suggested that inhibition of STAT3 activation was related with the reduction of IL17 and inflammation. ${ }^{22,23}$ Targeting STAT3 may be a plausible method for asthma treatment.
This current study aims to decipher the role of ADSCsderived exosomal miR-301a-3p in the pathogenesis of asthma. We discovered that ADSCs-derived exosomal miR-301a-3p suppressed the PDGF-BB-induced proliferation and migration of ASMCs, through downregulating the STAT3. These findings may shed light on the exploration of novel treatment for asthma.

\section{Materials and Methods}

\section{Clinical Specimen}

Blood samples of patients with asthma $(n=38)$ who hospitalized in First Affiliated Hospital of China Medical University from December 2019 to August 2020 were collected under the guidance and authorization of First Affiliated Hospital of China Medical University. Blood samples from healthy donors $(n=38)$ were enrolled as control. All participants have signed the written consents before the study and were informed about the purpose of the study. The studies involving human participants were reviewed and approved by First Affiliated Hospital of China Medical University, and were conducted in accordance with the Declaration of Helsinki. The characteristics of clinical samples were shown (Table S1).

\section{Establishment of a Mouse Model with}

\section{Asthma}

Male BALB/c mice (6-8 weeks old, 18-24 g) were treated with ovalbumin (OVA, Sigma, A5503) to induce asthma. In brief, $20 \mathrm{~g}$ complete Freund' s adjuvant was emulsified in $1 \mathrm{mg}$ aluminum hydroxide (Sigma, 239186), and then administrated into each mouse at a total volume of $0.2 \mathrm{~mL}$ on the 0,7 th, and 14 th day through intraperitoneal injection to sensitize the mice to OVA. On the 15th day, each mouse was exposed to 1\% OVA aerosol for 1 hour for 7 days. Mice in the control group were treated with normal saline instead. This study was carried out in accordance with the recommendations in the Guide for the Care and Use of Laboratory Animals of the National Institutes of Health. The protocol was approved by the Committee on the Ethics of Animal Experiments of the First Affiliated Hospital of China Medical University.

\section{Isolation and Identification of ADSCs}

ADSCs were isolated from the adipose tissue of healthy SD rats following a standard process. ${ }^{38}$ Cells were cultured in Dulbecco's Modified Eagle's medium (DMEM, Hyclone, USA) supplemented with $10 \%$ fetal bovine 
serum (FBS, Hyclone, USA), 1\% penicillin/streptomycin (Thermo, China) and L-glutamine ( $2 \mathrm{mM}$, Thermo). To identify the isolated ADSCs, we performed flow cytometry to detect the representative cell surface markers, including the CD31, CD45, CD90 and CD105. In brief, cells were hatched with fluorescence-labelled antibodies the markers, or IgG as negative control, followed by detection on FACS Calibur (BD Biosciences, USA).

\section{Isolation of ASMCs}

The ASMCs were isolated using a previously described protocol. ${ }^{39}$ In short, tracheas were obtained from healthy rats after anesthetization and death. The epithelium fibrous tissue was stripped, and remaining tissues were digested with elastase IV and collagenase I for 30 minutes at $37^{\circ} \mathrm{C}$. After that, the digested ASMCs were obtained by centrifuge, and incubated in DMEM complete medium.

\section{Isolation and Characterization of Exosomes}

The exosomes were isolated from the culture medium of ADSCs using a standard sequentially centrifuge method, ${ }^{14}$ and suspended in PBS at a density of $1 \mathrm{mg} / \mathrm{mL}$ for following experiments. Biomarkers of exosomes including CD63 and CD9 were detected by Western blotting assay. The size distribution of exosomes was determined by using Nanoparticle Tracking Analysis (NTA) on a Malvern's NanoSight (Germany).

\section{Exosome Internalization}

To observe the internalization of exosomes, we probed the ADSCs-derived exosomes by DiLC $_{18}$ (Sigma, USA) following manufacturer's instruction. In short, ADSCs were hatched with DiL-labelled exosomes (DiL-exo) for 24 hours, then the nuclei were dyed by DAPI reagent (Sigma). The ADSCs hatched with PBS were used as control. Images were taken by Carl Zeiss confocal microscope (USA).

\section{Cell Transfection}

The miR-301a-3p mimics, inhibitors, and negative control (NC), as well as STAT3 overexpressing plasmids pcDNASTAT3 (short as STAT3 OE) were purchased from QIAGEN (USA). Lipofectamine 2000 (Invitrogen) was adopted for cell transfection following manufacturer's instruction. In short, cells were seeded in 6-well plates, incubated with a mixture of $5 \mu \mathrm{L}$ Lipofectamine 2000 and $0.5 \mu \mathrm{g}$ oligonucleotides for 48 hours.

\section{Cell Viability and Apoptosis}

To determine cell viability, ASMCs were administrated with the cell counting kit-8 (CCK-8, Beyotime, China). Briefly, cells transfected with indicated oligonucleotides were placed in 96-well plates with 3000 cells in each well and treated with PDGF-BB for 48 hours. Subsequently, cells were disposed with CCK-8 reagent (10 ul) for one hour in the cell culturing incubator. Absorbance at $450 \mathrm{~nm}$ was measured by a spectrophotometric detector (Thermo). Cell apoptosis was evaluated by using an Annexin V/PI detection kit (Beyotime) following manufacturer's description.

\section{Transwell Migration Assay}

The migration ability of ASMCs was investigated by the Transwell assay with Matrigel-coated chambers (Costar, USA) with $8 \mu \mathrm{m}$ pore size. After suggested treatment, ASMCs suspension with FBS free DMEM $(200 \mu \mathrm{L})$ was placed in upper chamber, while the lower chamber was added with complete DMEM. Cells were incubated for 48 hours at the $37^{\circ} \mathrm{C}$ incubator, followed by staining with crystal violet for 20 minutes.

\section{Western Blotting and ELISA}

The total proteins were extracted from cells or exosomes by homogenizing with RIPA lysis buffer (Sigma) added with protease inhibitors (Sigma), and quantified by BCA method (Beyotime). An equal amount of proteins were separated by SDS-PAGE and shifted to PVDF membranes. Subsequently, the interested proteins were probed by specific primary antibodies against CD63, CD9, PCNA, MMP-9, and STAT3 overnight at $4{ }^{\circ} \mathrm{C}$. Next day, the protein bands were visualized by corresponding secondary antibodies and an ECL solution (Millipore, USA). All antibodies were obtained from Abcam and used following the instructions. The levels of cytokine released by ASMCs, including the tumor necrosis factor- $\alpha$ (TNF- $\alpha$ ), interleukin-1 $\beta$ (IL-1 $\beta$ ), and IL-6, were detected by Enzyme-linked immuno sorbent assay (ELISA) with commercial kits brought from Thermo in line with the manufacturer's protocols.

\section{Real-Time PCR Assay}

To quantify the levels of miR-301a-3p and STAT3 in ASMCS, ADSCs and exosomes, total RNAs were extracted by using Trizol reagent (Beyotime), reverse-transcribed to cDNA by using Easy Script kit (TransGen, China), and subjected to real-time PCR with a SYBR Green SuperMix 
kit (TransGen). The quantification of miR-301a-3p and STAT3 was normalized to internal control $\beta$-actin and U6, respectively. Primers were listed in Table S2.

\section{Luciferase Reporter Gene Assay}

TargetScan website was used for prediction of binding site between miR-301a-3p and the 3'UTR region of STAT3. The wild type (STAT3-WT) and mutated (STAT3-MUT) sequences of 3'UTR region of STAT3 were cloned to pGL3 control vectors, and co-transfected with miR-301a-3p mimics or negative control into ASMCs or 293T cells by using Lipofectamine 2000. pRL-TK vectors were co-transfected into cells as the internal control. Forty-eight hours after transfection, cells were lysed and a dual-luciferase reporter system (Promega, USA) was established to detect the luciferase activity.

\section{Statistical Analysis}

All data were shown as means with standard deviation (SD) of three replicates and analyzed by using a SPSS 19.0 software. The statistical analysis between two groups or multiple groups was evaluated by two-tailed unpaired Student's $t$-test or the one-way ANOVA analysis followed by a post hoc Tukey's test. $P<0.05$ was set as standard of statistical significance.

\section{Results}

\section{ADSCs-Derived Exosomes Regulated PDGF-BB-Stimulated Proliferation, Migration, Apoptosis and Inflammatory Responses of ASMCs}

To investigate the functions of ADSCs-derived exosomes on airway inflammation and remodeling, we extracted exosomes from ADSCs through a standard sequentially centrifuge method, and defined their functions in a PDGFBB-stimulated cell model. We first isolated ADSCs from the adipose of SD rats, verified by the negative expression of endothelial biomarkers CD31 and CD45, and the positive expression of mesenchymal stem cell markers CD90 and CD105 (Figure 1A). The notably elevated protein levels of CD63 and CD9 (Figure 1B), as well as the particle size around $100 \mathrm{~nm}$ (Figure 1C), suggested the successful isolation of exosomes from ADSCs. Besides, we testified the ability of ASMCs to uptake the ADSCs-derived exosomes. The appearance of DiL-labelled exosomes in plasma of ASMCs comparing with the control cells indicated the effective internalization of exosomes (Figure 1D). We next evaluated whether ADSCs-derived exosomes affect airway remodeling and inflammation. PDGF-BB treatment notably stimulated the proliferation (Figure $2 \mathrm{~A}$ ) and migration (Figure 2B) of ASMCs, whereas administration of ADSCsderived exosomes effectively alleviated these functions of PDGF-BB. Decreased expression of PCNA and MMP-9, the biomarkers of cell proliferation and migration, also supported the suppressed cell viability and migration after treatment of ADSCs-derived exosomes (Figure 2C and D). ADSCs-derived exosomes also promoted the apoptosis of ASMCs under stimulation of PDGF-BB (Figure 2E). Furthermore, the upregulated levels of inflammatory factors, including TNF- $\alpha$, IL- $1 \beta$ and IL-6, in ASMCs by PDGF-BB stimulation were notably decreased by the exosomes isolated from ADSCs. Above results indicated that ADSCs-derived exosomes played suppressive role during PDGF-BBstimulated inflammation and remodeling of ASMCs (Figure 2F-H).

\section{MiR-30la-3p Could Be Delivered from ADSCs to ASMCs by Exosomes}

We next tired to explore whether miR-301a-3p was involved in the function of ADSCs-derived exosomes on ASMCs against PDGF-BB stimulation. We treated the extracted exosomes with RNase A with or without Triton X100, and found that co-treatment of two agents notably decreased the levels of miR-301a-3p comparing with the control group, whereas RNase A alone did not alter the level of miR-301a-3p (Figure 3A). This finding suggested that there exists endogenous miR-301a-3p wrapped by the exosomes derived from ADSCs. Moreover, transfection with exogenous miR-301a-3p mimics notably elevated the levels of miR-301a-3p in both ADSCs and the secreted exosomes (Figure 3B). Administration of exosomes from miR-301a-3p transfected ADSCs also upregulated the level of miR-301a$3 p$ internalized by ASMCs (Figure 3C). Consistently, transfection with miR-301a-3p inhibitors decreased the levels of miR-301a-3p in ADSCs, the secreted exosomes (Figure 3D), and ASMCs (Figure 3E). Noteworthy, analysis on blood samples collected from healthy donors and patients with asthma revealed decreased level of miR301a-3p. These findings demonstrated that miR-301a-3p could be delivered from ADSCs to ASMCs through exosomes, and may function as a diagnostic biomarker for asthma (Figure 3F). 
A
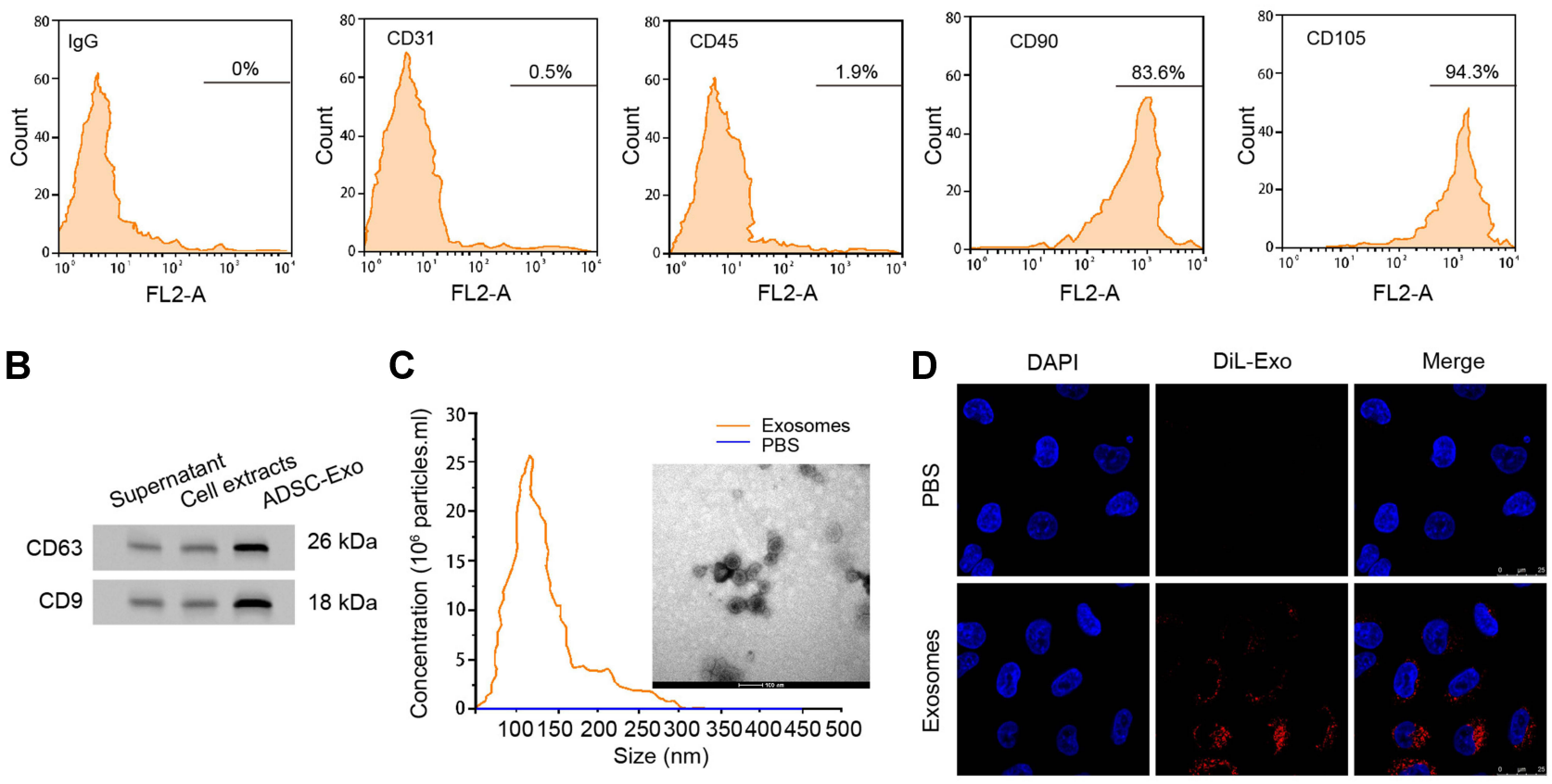

Figure I Exosomes derived from ADSCs could be internalized by ASMCs. (A) Flow cytometry to detect the expression of CD3I, CD45, CD90, CDI05, and negative control lgG on surface of ADSCs. (B) Western blotting experiment to detect the protein markers CD63 and CD9 in supernatant, cell extracts or exosomes from ADSCs. (C) Size distribution of exosomes was measured by Nanoparticle Tracking Analysis (NTA). (D) Exosomes isolated from ADSCs were labelled by DiLCI8 (DiL) and administrated to ASMCs for 24 hours, fluorescence was detected by confocal experiment.

\section{Exosomal miR-30la-3p Regulated PDGF-BB-Stimulated Proliferation, Migration, Apoptosis and Inflammation of ASMCs}

Under the stimulation of PDGF-BB, exosomal miR-301a$3 \mathrm{p}$ could notably reverse the elevated proliferation (Figure 4A) and migration (Figure 4B and $\mathrm{C}$ ) of ASMCs, along with decreased expression of PCNA and MMP-9 (Figure 4D). The suppressed apoptosis of ASMCs under PDGF-BB stimulation could also be remarkably relieved by exosomal miR-301a-3p (Figure 4E). On the other hand, inflammatory response caused by PDGF-BB stimulation was suppressed by exosomal miR-301a-3p, manifested by decreased release of TNF- $\alpha$, IL-1 $\beta$ and IL-6 (Figure 4F-H). These data manifested that exosomal miR-301a-3p regulated PDGFBB-stimulated proliferation, migration, apoptosis and inflammation of ASMCs.

\section{MiR-30la-3p Regulated Functions of ASMCs Through Interacting with STAT3}

To elucidate the mechanism underlying the specific role of exosomal miR-301a-3p in ASMCs, we conducted target prediction via online website TargetScan and spotted STAT3 as a potential target of miR-301a-3p (Figure 5A). Next, we conducted luciferase reporter assay to verify the direct interaction between miR301a-3p with the 3'UTR region of STAT3. As shown in Figure 5B, transfection of miR-301a-3p mimics notably suppressed the luciferase activity of the reporter gene plasmids containing wild-type STAT3 (WT) in comparison with $\mathrm{NC}$ mimics transfection, whereas the activity of mutated STAT3 reporter plasmids was not significantly altered, in both ASMCs and 293T cells. The efficacy of miR-301a-3p transfection was determined by qRT-PCR assay (Figure 5C). The mRNA (Figure 5D) and protein (Figure 5E) levels of STAT3 were remarkably decreased after transfection with miR301a-3p mimics. Moreover, the expression of STAT3 in specimen collected from patients with asthma was notably higher than that of the normal control (Figure 5F), and exhibited a negative correlation with expression of miR-301a-3p (Figure 5G). Further functional analyses explored the regulatory role of miR-301a-3p/STAT3 axis in PDGF-BB-stimulated ASMC model. We found that the suppressed proliferation and migration of ASMCs under treatment of 

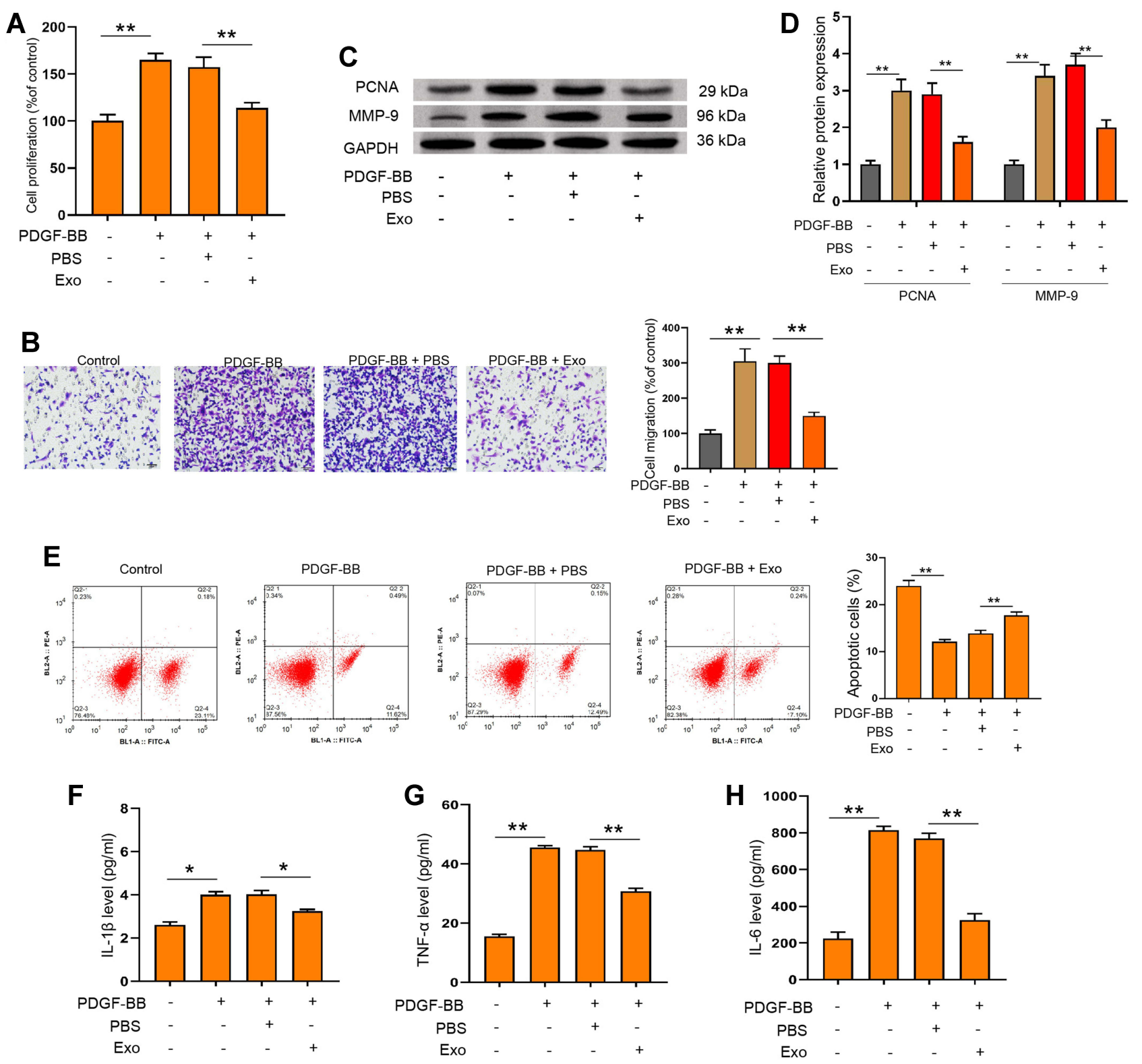

Figure 2 ADSCs-derived exosomes suppressed PDGF-BB-stimulated proliferation, migration, apoptosis and inflammation of ASMCs. (A and B) ASMCs were treated with ADSCs-derived exosomes with or without PDGF-BB stimulation, then cell viability was determined by CCK-8 experiment. (C-H) ASMCs were treated with ADSCsderived exosomes under PDGF-BB stimulation. Cell migration, protein levels of PCNA and MMP-9, and apoptosis were measured by Transwell (C) Western blotting (D) and flow cytometry $(\mathbf{E})$. The secretion of IL-I $\beta(\mathbf{F})$ IL-6 $(\mathbf{G})$ and TNF- $\alpha(\mathbf{H})$ were evaluated by ELISA assay. Exo: ADSCs-derived exosomes. $* p<0.05 ; * * p<0.0$ I.

exosomal miR-301a-3p were notably enhanced by ectopic expression of STAT3 (Figure 6A and B). Besides, STAT3 overexpression could recover the PDGF-BB-suppressed apoptosis of ASMCs under administration of exosomal miR-301a-3p (Figure 6C). The decreased release of inflammatory cytokines TNF$\alpha$, IL-1 $\beta$ and IL- 6 were by exosomal miR-301a-3p was also significantly alleviated by STAT3 overexpression (Figure 6D-F). Collectively, these data suggested that STAT3 acts as a target of miR-301a-3p to mediate the remodeling and inflammation of ASMCs under PDGFBB stimulation.

\section{Exosomal miR-30la-3p Inhibits OVA-Induced Lung Injury by Targeting STAT3 in Mice}

We then observed that the fibrosis of OVA mice was attenuated by the treatment of exosomal miR-301a-3p in the model (Figure 7A). Meanwhile, we observed that the 
A

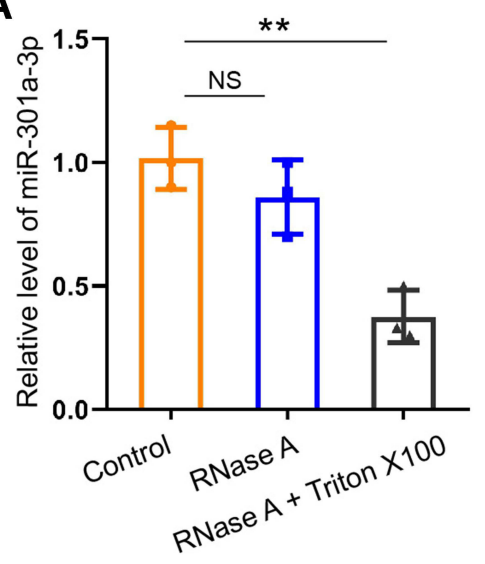

D

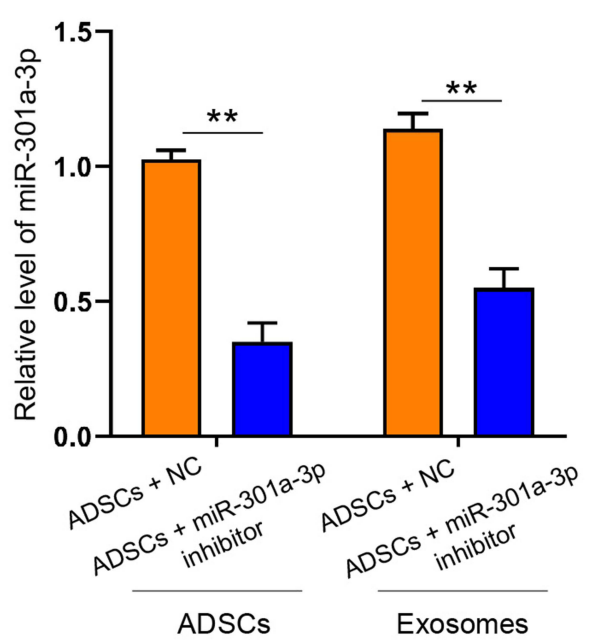

B

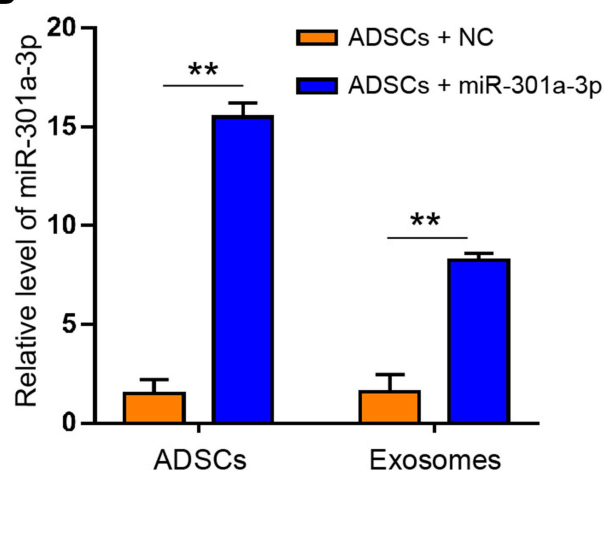

C

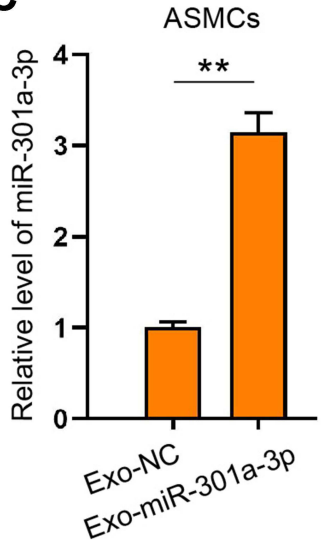

E

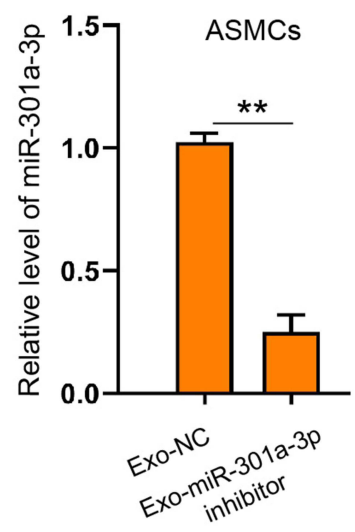

F

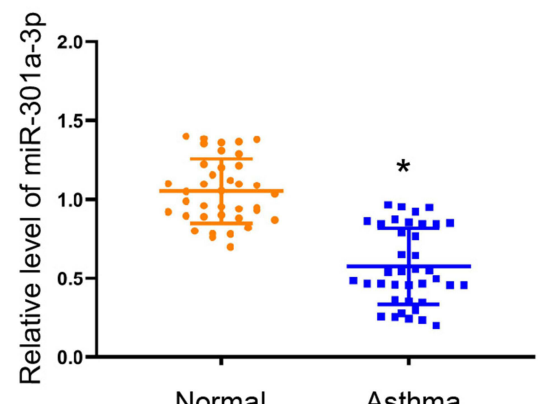

Figure 3 MiR-30la-3p could be delivered by exosomes to ASMCs. (A) Exosomes derived from ADSCs were treated with RNase A with or without Triton XI00, then the level of miR-30la-3p was measured by qRT-PCR. (B) Level of miR-30la-3p in ADSCs and the secreted exosomes after transfection with miR-30 la-3p mimics were measured by qRT-PCR. (C) Level of miR-30la-3p in ASMCs after treatment with exosomes-derived from transfected ADSCs were measured by qRT-PCR. (D and E) ADSCs were transfected with miR-30la-3p inhibitors or negative control (NC), and exosomes were isolated for treatment of ASMCs. The level of miR-30la-3p in ADSCs and the secreted exosomes (D), and the ASMCs (E) were measured by qRT-PCR. (F) Level of miR-30la-3p in blood samples collected from healthy donors (Normal) and patients with asthma were detected by qRT-PCR. Exo-miR-30la-3p: exosomes extracted from ADSCs after transfection with miR-30la-3p mimics; Exo-miR-30la-3p inhibitor: exosomes extracted from ADSCs after transfection with miR-30 Ia-3p inhibitor; Exo-NC: exosomes extracted from ADSCs after transfection with negative control mimics. $*_{p}<0.05 ; *_{p}<0.01$.

levels of IL-1 $\beta$, IL-6, TNF- $\alpha$, and MCP-1 were induced in the OVA mice, in which the treatment of exosomal miR$301 \mathrm{a}-3 \mathrm{p}$ reversed the levels in the model (Figure 7B-E).

\section{Discussion}

The inflammation and remodeling of airway are the most critical processes of asthma, which is characterized by activated proliferation and migration of ASMCs., $4,24,25$ Platelet-derived growth factor (PDGF)-BB is a member of PDGF family, which has been proven to act as a driver for phenotype switch of ASMCs. ${ }^{11,26}$ In this work, we adopted PDGF-BB for establishment of cell model to mimic remodeling and inflammation of ASMCs, which manifested as elevated proliferation, migration and levels of inflammatory factors, along with decreased cell apoptosis.

Over the past decades, MSCs have presented great potential for treatment of disease including asthma. ${ }^{12}$ It was previously reported that transplantation of MSCs benefited the improvement of airway inflammation in asthmalike circumstance. ${ }^{27,28}$ MSCs are generally derived from bone marrow and some other tissues such as adipose, and the regulatory effects of MSCs largely rely on the release of soluble molecules and the secretion of extracellular vesicles such as exosomes. ${ }^{29}$ The functions of ADSCs-derived exosomes are also widely reported during various cellular processes. ADSCs-derived exosomes could regulate the proliferation, migration, and apoptosis of breast cancer 
A

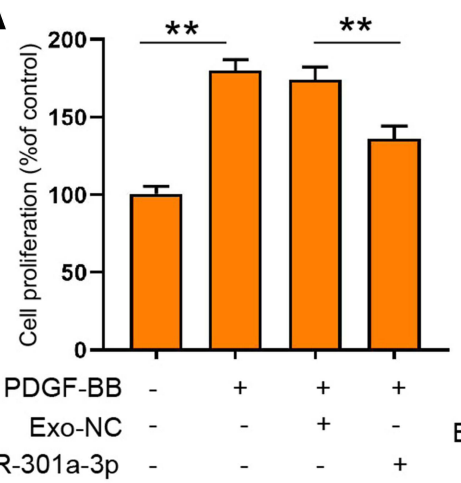

\section{B}

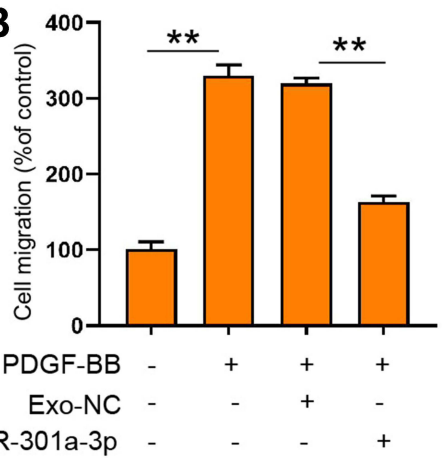

C

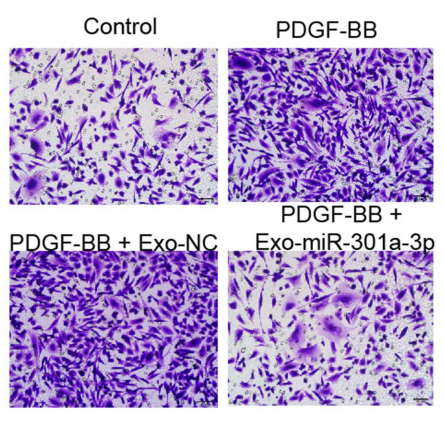

Exo-miR-301a-3p

D
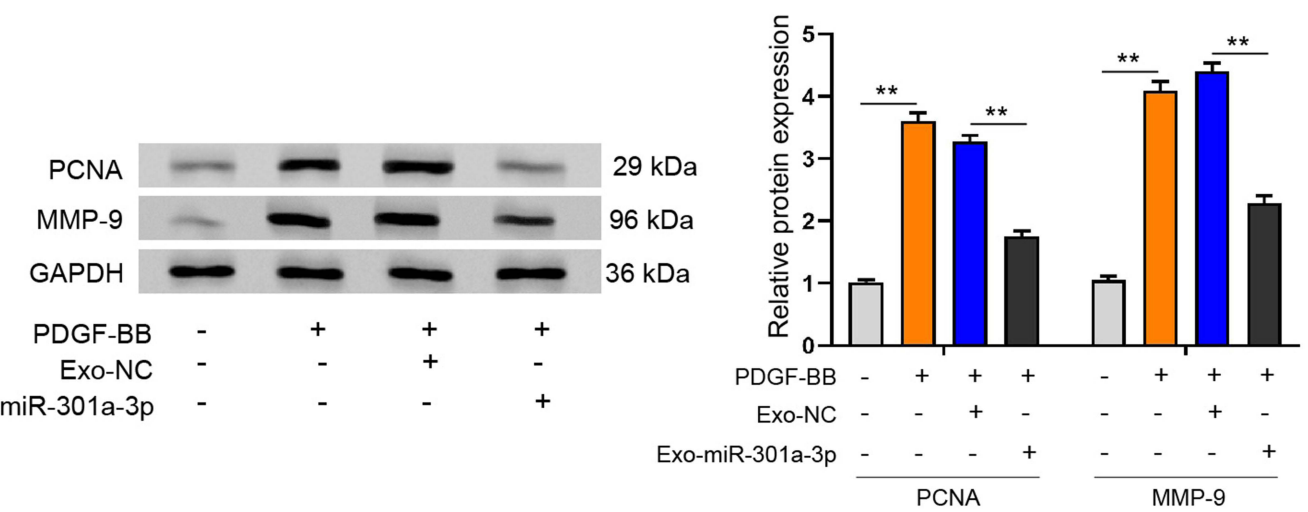

E

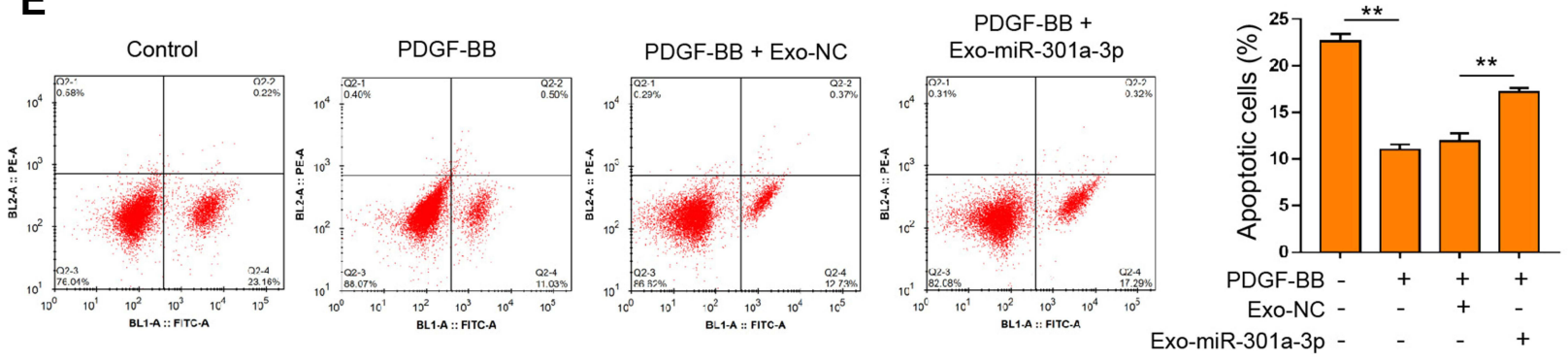

F

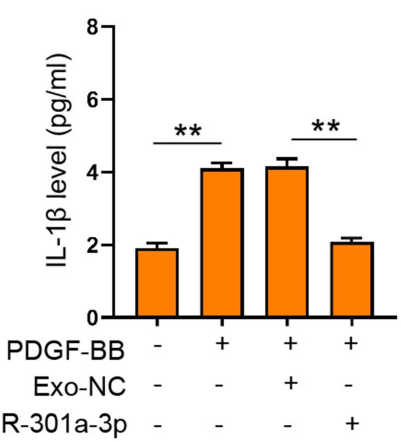

G

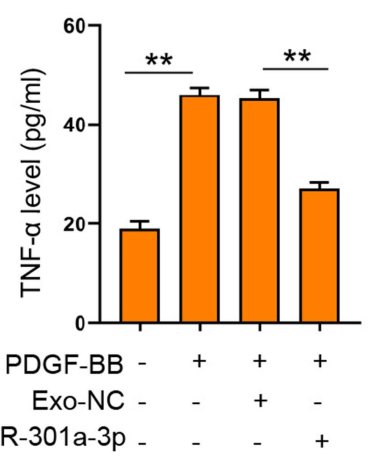

H

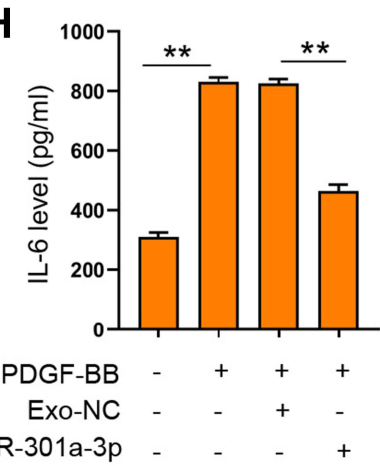

Figure 4 Exosomal miR-30la-3p suppressed PDGF-BB-stimulated proliferation, migration, apoptosis and inflammation of ASMCs. (A and B) ASMCs were treated with exosomal miR-30 la-3p with or without PDGF-BB stimulation, then cell viability was determined by CCK-8 experiment. (C-H) ASMCs were treated with exosomes isolated from ASMCs after transfection with miR-30la-3p mimics (exosomal miR-30la-3p) or negative control (exosomal NC) under PDGF-BB stimulation. Cell migration, protein levels of PCNA and MMP-9, and apoptosis were measured by Transwell (C) Western blotting (D) and flow cytometry (E). The secretion of IL-I $\beta$ (F) IL-6 (G) and TNF- $\alpha$ (H) were evaluated by ELISA assay. ${ }^{* *} p<0.01$. 
A

miR-301a-3p 3'-CGAAACUGUUAUGAUAACGUGAC-5'

|| || || |

STAT3-WT 5'-UACAUACUCCUGGCAUUGCACUU-3'

STAT3-MUT 5'-UACAUACUCCUGGCAUACACGAU-3'
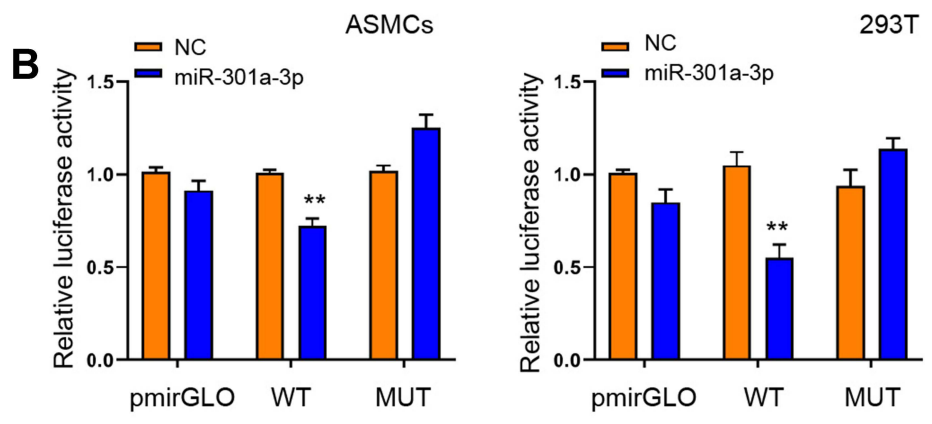

C

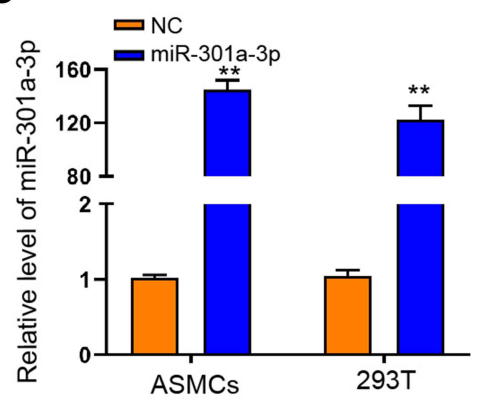

$\mathbf{F}$

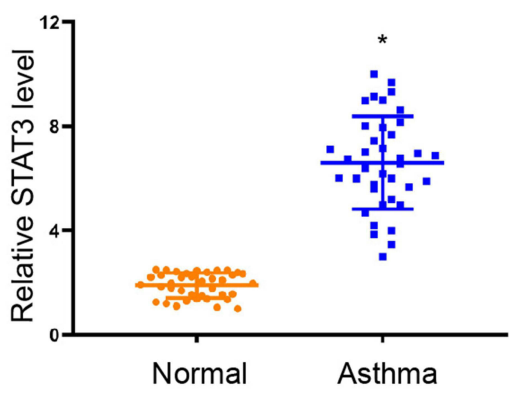

D

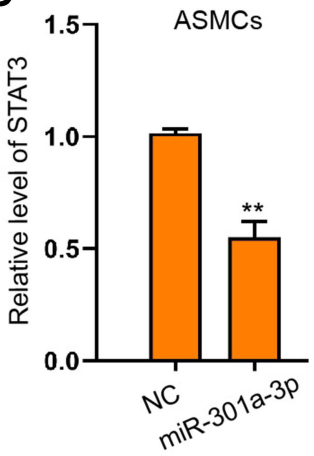

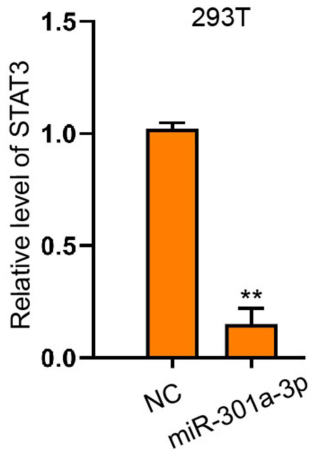

E

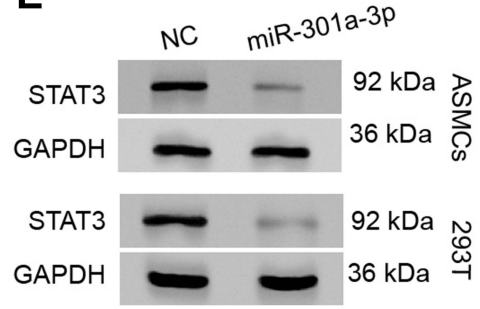

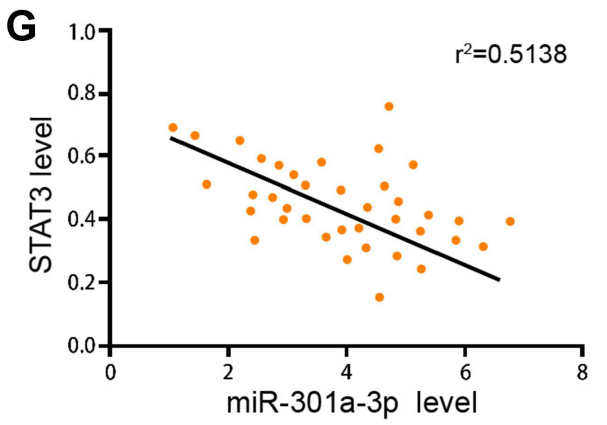

Figure 5 MiR-30 la-3p interacted with STAT3 to suppress its expression in ASMCs. (A) TargetScan online tool prediction of interaction between miR-30 Ia-3p with STAT3, and the mutated sequence of STAT3 3'UTR. (B) Luciferase activity of STAT3-WT and STAT3-MUT under transfection with miR-30 Ia-3p mimics or negative control (NC). (C) QRT-PCR assay was performed to detect the level of miR-30la-3p in ASMCs and 293T cells under transfection of miR-30la-3p or NC. (D and E) QRT-PCR and Western blotting assay to determine the mRNA (D) and protein (E) level of STAT3 in ASMCs and 293T cells under transfection of miR-30 la-3p or NC. (F) Relative level of STAT3 in patients with asthma and healthy donors (Normal). (G) Pearson correlation analysis of miR-30la-3p and STAT3. * $p<0.05$; ${ }^{* *} p<0.0$ I.

cells and vascular endothelial cell and so on. ${ }^{30-32}$ As for the regulation of immune and inflammatory responses, ADSCsderived exosomes impede differentiation of $\mathrm{T}$ cells toward effector or memory cell phenotypes, and reduced the release of interferon-gamma (IFN- $\gamma$ ). ${ }^{33}$ Administration of ADSCsderived exosomes are indicated to ameliorate inflammatory responses and suppressed secretion of inflammatory cytokines, including IL-4, IL-23, and TNF- $\alpha$, in a mouse model of atopic dermatitis-like symptoms. ${ }^{34}$ Our work demonstrated that exosomes extracted from ADSCs impeded the PDGF-BB-stimulated proliferation and migration of ASMCs, decreased the secretion of inflammatory factors TNF- $\alpha$, IL-1 $\beta$ and IL-6.
The functions of exosomes on cell communication are closely correlated with their ability to deliver various signaling molecules, especially the miRNAs. ${ }^{16}$ ExosomalmiRNAs such as let-7a, miRNA-24, and miRNA-200c, showed substantially different levels between patients with asthma and the healthy subjects. ${ }^{35}$ Treatment with GW4869, the inhibitor of exosome generation, impeded eosinophil deposition in mucosa and airway walls. ${ }^{36}$ In present study, further evaluation of miRNA in ADSCs-derived exosomes suggested existence of exosomal miR-301a-3p, and decreased level of miR-301a-3p in blood samples collected from asthma patients comparing with the healthy subjects. The exosomal miR-301a-3p effectively counteracted the 

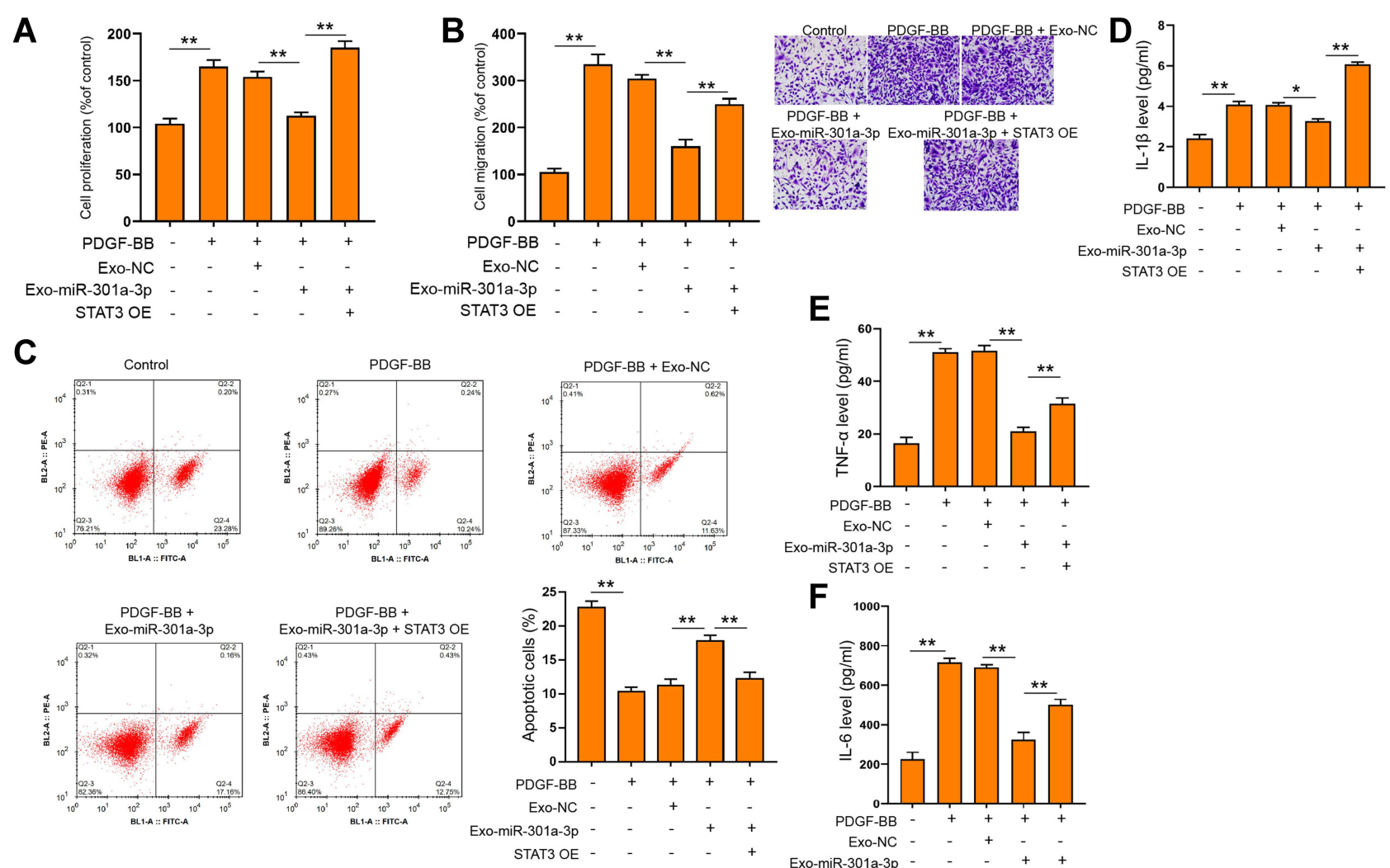

E

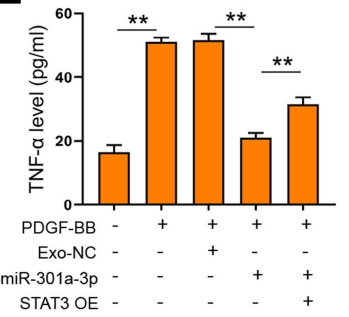

$\mathbf{F}$

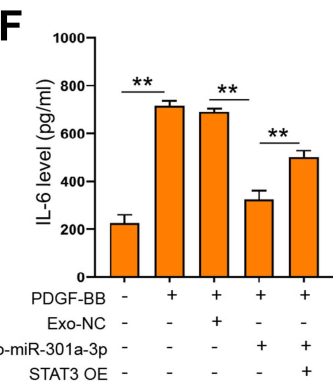

Figure 6 Exosomal miR-30la-3p regulated PDGF-BB-stimulated functions of ASMCs via targeting STAT3. ASMCs were treated with exosomes isolated from ASMCs after transfection with miR-30la-3p mimics (exosomal miR-30la-3p) or negative control (exosomal NC), and ectopic expression of STAT3 (STAT3 OE) under PDGF-BB stimulation. Cell viability, migration, and apoptosis were measured by CCK-8 (A) Transwell (B) and flow cytometry (C). The secretion of IL-I $\beta$ (D) IL-6 (E) and TNF- $\alpha$ (F) were evaluated by ELISA assay. $*_{p}<0.05 ; * *_{p}<0.01$.

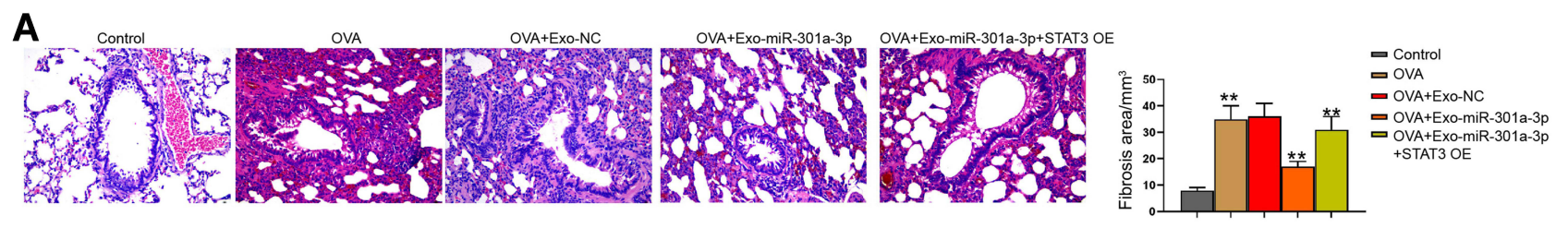

B

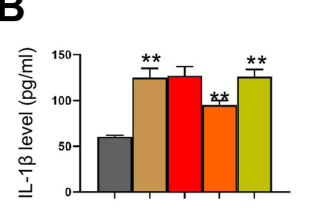

D

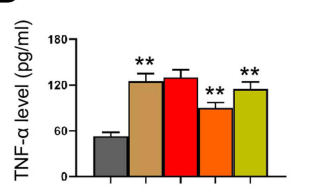

= Control

- OVA

- OVA+Exo-NC

- OVA+Exo-miR-301a-3p

OVA+Exo-miR-301a-3p

STAT3 OE
C

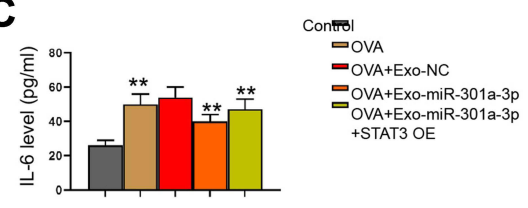

E

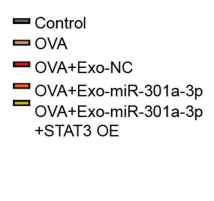

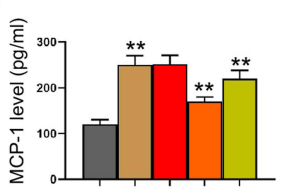

- Control

口OVA

= OVA+Exo-NC

OVA+Exo-miR-301a-3p

OVA+EXo-miR-301a-3p
+ STAT3 OE

Figure 7 Exosomal miR-30la-3p inhibits OVA-induced lung injury by targeting STAT3 in mice. (A) Fibrosis in mouse lung tissues was measured by Masson's trichrome staining. (B-E) Secretion of IL-I $\beta$, IL-6, TNF- $\alpha$, and MCP-I was measured by ELISA kits. $n=5$. ** $p<0.01$.

PDGF-BB-stimulated remodeling and inflammatory response of ASMCs. Subsequently, we identified STAT3 as a target of miR-301a-3p in ASMCs, and the negatively correlated expression between STAT3 and miR-301a-3p in blood samples collected from patients with asthma. Noteworthy, the role of STAT3 in lung inflammation is 
quite controversial. Several studies suggested that inhibition of STAT3 activity was correlated with the reduction of IL-17 and inflammation. ${ }^{22,23}$ Moreover, STAT-3 delivered by ADSC-derived exosomes induced the polarization of antiinflammatory M2 macrophage through the transactivation of arginase-1 and significantly suppressed inflammatory responses caused by lipopolysaccharide (LPS) and IFN- $\gamma .{ }^{37}$ Targeting STAT3 is thereby a potential therapeutic method for asthma. There were still some limitations in this study. The difference of exosomes in serum cannot represent the difference of local microenvironment. Meanwhile, the relationship between exosomes and airway remodeling in asthmatic patients is needed to be explored in future investigations.

\section{Conclusion}

In summary, this research deciphered the inhibitory function of ADSC-derived exosomal miR-301a-3p on ASMCs, exploited the STAT3 as a target of miR-301a-3p during this process. Our work presented ADSC-derived exosomal miR-301a-3p as a novel and effective treatment for asthma.

\section{Funding}

This work was supported by projects from The 13th FiveYear Plan National Key R\&D Program for Precision Medicine Research (No.: 2016YFC1304500), The National Nature Science of Foundation of China (No.: 81770021).

\section{Disclosure}

The authors report no conflicts of interest in this work.

\section{References}

1. Lemanske RF Jr., Busse WW. Asthma. JAMA. 1997;278 (22):1855-1873. doi:10.1001/jama.1997.03550220061010

2. Mims JW. Asthma: definitions and pathophysiology. Int Forum Allergy Rhinol. 2015;5(Suppl 1):S2-6. doi:10.1002/alr.21609

3. Sockrider M, Fussner L. What is asthma? Am J Respir Crit Care Med. 2020;202(9):PP25-P26. doi:10.1164/rccm.2029P25

4. Bousquet J, Mantzouranis E, Cruz AA, et al. Uniform definition of asthma severity, control, and exacerbations: document presented for the World Health Organization consultation on severe asthma. J Allergy Clin Immunol. 2010;126(5):926-938. doi:10.1016/j.jaci.20 10.07.019

5. Poon AH, Hamid Q. Severe asthma: Have we made progress? Ann Am Thorac Soc. 2016;13(Suppl 1):S68-77. doi:10.1513/AnnalsATS.20 1508-514MG

6. Bhalla A, Mukherjee M, Nair P. Airway eosinophilopoietic and autoimmune mechanisms of eosinophilia in severe asthma. Immunol Allergy Clin North Am. 2018;38(4):639-654. doi:10.1016/j.iac.20 18.06.003
7. Liang X, Wang J, Chen W, et al. Inhibition of airway remodeling and inflammation by isoforskolin in PDGF-induced rat ASMCs and OVA-induced rat asthma model. Biomed Pharmacother. 2017;95: 275-286. doi:10.1016/j.biopha.2017.08.063

8. Mouraux S, Bernasconi E, Pattaroni C, et al. Airway microbiota signals anabolic and catabolic remodeling in the transplanted lung. J Allergy Clin Immunol. 2018;141(2):718-729 e717. doi:10.1016/j. jaci.2017.06.022

9. Castillo JR, Peters SP, Busse WW. Asthma exacerbations: pathogenesis, prevention, and treatment. J Allergy Clin Immunol Pract. 2017;5 (4):918-927. doi:10.1016/j.jaip.2017.05.001

10. Hartley R, Berair R, Brightling CE. Severe asthma: novel advances in the pathogenesis and therapy. Pol Arch Med Wewn. 2014;124 (5):247-254. doi:10.20452/pamw.2253

11. Halayko AJ, Tran T, Gosens R. Phenotype and functional plasticity of airway smooth muscle: role of caveolae and caveolins. Proc Am Thorac Soc. 2008;5(1):80-88. doi:10.1513/pats.200705$057 \mathrm{VS}$

12. Zhu W, Huang L, Li Y, et al. Mesenchymal stem cell-secreted soluble signaling molecules potentiate tumor growth. Cell Cycle. 2011;10 (18):3198-3207. doi:10.4161/cc.10.18.17638

13. Kariminekoo S, Movassaghpour A, Rahimzadeh A, Talebi M, Shamsasenjan K, Akbarzadeh A. Implications of mesenchymal stem cells in regenerative medicine. Artif Cells Nanomed Biotechnol. 2016;44(3):749-757. doi:10.3109/21691401.2015.1129620

14. Wu X, Showiheen SAA, Sun AR, et al. Exosomes extraction and identification. Methods Mol Biol. 2019;2054:81-91.

15. Kalluri R. The biology and function of exosomes in cancer. $J$ Clin Invest. 2016;126(4):1208-1215. doi:10.1172/JCI81135

16. Zhang L, Yu D. Exosomes in cancer development, metastasis, and immunity. Biochim Biophys Acta Rev Cancer. 2019;1871(2): 455-468. doi:10.1016/j.bbcan.2019.04.004

17. Wortzel I, Dror S, Kenific CM, Lyden D. Exosome-mediated metastasis: communication from a distance. Dev Cell. 2019;49(3):347-360. doi:10.1016/j.devcel.2019.04.011

18. Qu Y, Zhang Q, Cai X, et al. Exosomes derived from miR-181-5pmodified adipose-derived mesenchymal stem cells prevent liver fibrosis via autophagy activation. $J$ Cell Mol Med. 2017;21(10): 2491-2502. doi:10.1111/jcmm.13170

19. Wang X, Luo G, Zhang K, et al. Hypoxic Tumor-Derived Exosomal miR-301a Mediates M2 Macrophage Polarization via PTEN/PI3Kgamma to Promote Pancreatic Cancer Metastasis. Cancer Res. 2018;78(16):4586-4598. doi:10.1158/0008-5472. CAN-17-3841

20. Xia X, Wang S, Ni B, et al. Hypoxic gastric cancer-derived exosomes promote progression and metastasis via MiR-301a-3p/PHD3/HIF1alpha positive feedback loop. Oncogene. 2020;39(39):6231-6244. doi:10.1038/s41388-020-01425-6

21. Hillmer EJ, Zhang H, Li HS, Watowich SS. STAT3 signaling in immunity. Cytokine Growth Factor Rev. 2016;31:1-15. doi:10.1016/ j.cytogfr.2016.05.001

22. Gavino AC, Nahmod K, Bharadwaj U, Makedonas G, Tweardy DJ. STAT3 inhibition prevents lung inflammation, remodeling, and accumulation of Th2 and Th17 cells in a murine asthma model. Allergy. 2016;71(12):1684-1692. doi:10.1111/all.12937

23. Harris TJ, Grosso JF, Yen HR, et al. Cutting edge: an in vivo requirement for STAT3 signaling in TH17 development and TH17-dependent autoimmunity. $J$ Immunol. 2007;179(7):43 13-4317. doi:10.4049/jimmunol.179.7.4313

24. Bentley JK, Hershenson MB. Airway smooth muscle growth in asthma: proliferation, hypertrophy, and migration. Proc Am Thorac Soc. 2008;5(1):89-96. doi:10.1513/pats.200705-063VS

25. Prakash YS. Airway smooth muscle in airway reactivity and remodeling: what have we learned? Am J Physiol Lung Cell Mol Physiol. 2013;305(12):L912-933. doi:10.1152/ajplung.002 59.2013 
26. Hoshino M, Takahashi M, Aoike N. Expression of vascular endothelial growth factor, basic fibroblast growth factor, and angiogenin immunoreactivity in asthmatic airways and its relationship to angiogenesis. J Allergy Clin Immunol. 2001;107(2):295-301. doi:10. 1067/mai.2001.111928

27. Kang SY, Park DE, Song WJ, et al. Immunologic regulatory effects of human umbilical cord blood-derived mesenchymal stem cells in a murine ovalbumin asthma model. Clin Exp Allergy. 2017;47 (7):937-945. doi:10.1111/cea.12920

28. Lau AN, Goodwin M, Kim CF, Weiss DJ. Stem cells and regenerative medicine in lung biology and diseases. Mol Ther. 2012;20 (6):1116-1130. doi:10.1038/mt.2012.37

29. Lo Sicco C, Reverberi D, Balbi C, et al. Mesenchymal stem cell-derived extracellular vesicles as mediators of anti-inflammatory effects: endorsement of macrophage polarization. Stem Cells Transl Med. 2017;6(3):1018-1028. doi:10.1002/sctm.16-0363

30. Lin KC, Yip HK, Shao PL, et al. Combination of adipose-derived mesenchymal stem cells (ADMSC) and ADMSC-derived exosomes for protecting kidney from acute ischemia-reperfusion injury. Int J Cardiol. 2016;216:173-185. doi:10.1016/j.ijcard.2016.04.061

31. Ma T, Fu B, Yang X, Xiao Y, Pan M. Adipose mesenchymal stem cell-derived exosomes promote cell proliferation, migration, and inhibit cell apoptosis via Wnt/beta-catenin signaling in cutaneous wound healing. J Cell Biochem. 2019;120(6):10847-10854. doi:10.1002/ jcb. 28376

32. Gangadaran P, Rajendran RL, Lee HW, et al. Extracellular vesicles from mesenchymal stem cells activates VEGF receptors and accelerates recovery of hindlimb ischemia. $J$ Control Release. 2017;264:112-126. doi:10.1016/j.jconrel.2017.08.022
33. Blazquez R, Sanchez-Margallo FM, de la Rosa O, et al. Immunomodulatory potential of human adipose mesenchymal stem cells derived exosomes on in vitro stimulated T cells. Front Immunol. 2014;5:556. doi:10.3389/fimmu.2014.00556

34. Cho BS, Kim JO, Ha DH, Yi YW. Exosomes derived from human adipose tissue-derived mesenchymal stem cells alleviate atopic dermatitis. Stem Cell Res Ther. 2018;9(1):187. doi:10.1186/s13287018-0939-5

35. Levanen B, Bhakta NR, Torregrosa Paredes P, et al. Altered microRNA profiles in bronchoalveolar lavage fluid exosomes in asthmatic patients. J Allergy Clin Immunol. 2013;131(3):894-903. doi:10.1016/j.jaci.2012.11.039

36. Gon Y, Maruoka S, Inoue $\mathrm{T}$, et al. Selective release of miRNAs via extracellular vesicles is associated with house-dust mite allergen-induced airway inflammation. Clin Exp Allergy. 2017;47 (12):1586-1598. doi:10.1111/cea.13016

37. Zhao H, Shang Q, Pan Z, et al. Exosomes from adipose-derived stem cells attenuate adipose inflammation and obesity through polarizing M2 macrophages and Beijing in white adipose tissue. Diabetes. 2018;67(2):235-247. doi:10.2337/db17-0356

38. Yu S, Cheng Y, Zhang L, et al. Treatment with adipose tissue-derived mesenchymal stem cells exerts anti-diabetic effects, improves long-term complications, and attenuates inflammation in type 2 diabetic rats. Stem Cell Res Ther. 2019;10(1):333. doi:10.1186/s13287019-1474-8

39. Salter B, Pray C, Radford K, Martin JG, Nair P. Regulation of human airway smooth muscle cell migration and relevance to asthma. Respir Res. 2017;18(1):156. doi:10.1186/s12931-017-0640-8

\section{Publish your work in this journal}

The Journal of Asthma and Allergy is an international, peer-reviewed open-access journal publishing original research, reports, editorials and commentaries on the following topics: Asthma; Pulmonary physiology; Asthma related clinical health; Clinical immunology and the immunological basis of disease; Pharmacological interventions and new therapies. The manuscript management system is completely online and includes a very quick and fair peer-review system, which is all easy to use. Visit http://www.dovepress.com/testimonials.php to read real quotes from published authors. 